\title{
Long-term outcome after Gamma Knife radiosurgery for acoustic neuroma of all Koos grades: a single-center study
}

\author{
Josa M. Frischer, MD, PhD, ${ }^{1}$ Elise Gruber, ${ }^{1}$ Verena Schöffmann, ${ }^{1}$ Adolf Ertl, PhD, ${ }^{1}$ \\ Romana Höftberger, MD, ${ }^{2}$ Ammar Mallouhi, MD, ${ }^{3}$ Stefan Wolfsberger, MD, ${ }^{1}$ \\ Christoph Arnoldner, MD, ${ }^{4}$ Wilhelm Eisner, MD, ${ }^{5}$ Engelbert Knosp, MD, ${ }^{1}$ Klaus Kitz, MD, ${ }^{1}$ and \\ Brigitte Gatterbauer, MD'
}

Departments of ${ }^{1}$ Neurosurgery, ${ }^{3}$ Radiology, and ${ }^{4}$ Otorhinolaryngology; ${ }^{2}$ Institute of Neurology, Medical University Vienna; and ${ }^{5}$ Department of Neurosurgery, Medical University Innsbruck, Austria

OBJECTIVE The authors present long-term follow-up data on patients treated with Gamma Knife radiosurgery (GKRS) for acoustic neuroma.

METHODS Six hundred eighteen patients were radiosurgically treated for acoustic neuroma between 1992 and 2016 at the Department of Neurosurgery, Medical University Vienna. Patients with neurofibromatosis and patients treated too recently to attain 1 year of follow-up were excluded from this retrospective study. Thus, data on 557 patients with spontaneous acoustic neuroma of any Koos grade are presented, as are long-term follow-up data on 426 patients with a minimum follow-up of 2 years. Patients were assessed according to the Gardner-Robertson (GR) hearing scale and the House-Brackmann facial nerve function scale, both prior to GKRS and at the times of follow-up.

RESULTS Four hundred fifty-two patients (81\%) were treated with radiosurgery alone and 105 patients (19\%) with combined microsurgery-radiosurgery. While the combined treatment was especially favored before 2002, the percentage of cases treated with radiosurgery alone has significantly increased since then. The overall complication rate after GKRS was low and has declined significantly in the last decade. The risk of developing hydrocephalus after GKRS increased with tumor size. One case $(0.2 \%)$ of malignant transformation after GKRS was diagnosed. Radiological tumor control rates of $92 \%, 91 \%$, and $91 \%$ at 5, 10, and 15 years after GKRS, regardless of the Koos grade or pretreatment, were observed. The overall tumor control rate without the need for additional treatment was even higher at $98 \%$. At the last follow-up, functional hearing was preserved in $55 \%$ of patients who had been classified with GR hearing class I or II prior to GKRS. Hearing preservation rates of $53 \%, 34 \%$, and $34 \%$ at 5,10 , and 15 years after GKRS were observed. The multivariate regression model revealed that the GR hearing class prior to GKRS and the median dose to the cochlea were independent predictors of the GR class at follow-up.

CONCLUSIONS In small to medium-sized spontaneous acoustic neuromas, radiosurgery should be recognized as the primary treatment at an early stage. Although minimizing the cochlear dose seems beneficial for hearing preservation, the authors, like others before, do not recommend undertreating intracanalicular tumors in favor of low cochlear doses. For larger acoustic neuromas, radiosurgery remains a reliable management option with tumor control rates similar to those for smaller acoustic neuromas; however, careful patient selection and counseling are recommended given the higher risk of side effects. Microsurgery must be considered in acoustic neuromas with significant brainstem compression or hydrocephalus.

https://thejns.org/doi/abs/10.3171/2017.8.JNS171281

KEY WORDS Gamma Knife radiosurgery; acoustic neuroma; vestibular schwannoma; Koos grade; hydrocephalus; hearing preservation; stereotactic radiosurgery

ABBREVIATIONS Cl = cochlear implant; GKRS = Gamma Knife radiosurgery; GR = Gardner-Robertson; HB = House-Brackmann; NF2 = neurofibromatosis type 2; SRS = stereotactic radiosurgery.

SUBMITTED May 24, 2017. ACCEPTED August 21, 2017.

INCLUDE WHEN CITING Published online March 2, 2018; DOI: 10.3171/2017.8.JNS171281. 
$\mathrm{I}$ $\mathrm{N}$ recent years, radiosurgery has been established as a first-line treatment option for acoustic neuromas. Excellent tumor control rates have been reported in multiple large series. . $^{3,10,23,25,35}$ In addition, several studies have attested to a significantly lower postoperative complication rate for radiosurgery than for microsurgical resection. ${ }^{30,31}$ However, the literature includes continuing debates regarding the potential loss of hearing after radiosurgery and the associated risk factors, as well as the treatment of large acoustic neuromas. ${ }^{7,16,41}$ Moreover, for neurofibromatosis type 2 (NF2) patients with postlingual deafness, cochlear implants (CIs) have provided favorable hearing restoration, which has apparently reopened discussions about acoustic neuroma management even in patients with spontaneous acoustic neuroma. ${ }^{40}$ In the present study, we present long-term follow-up data on a large patient series, identify risk factors for hearing loss after radiosurgery for acoustic neuroma of any Koos grade, and describe changes in acoustic neuroma management over the last 2 decades at our institution.

\section{Methods}

\section{Patient Sample and Data Evaluation}

This study was approved by the ethics review committee of the Medical University Vienna. Six hundred eighteen patients were treated with Gamma Knife radiosurgery (GKRS) for acoustic neuroma between 1992 and 2016 at the Department of Neurosurgery, Medical University Vienna. Fifty-three patients had not attained 1 year of follow-up by the end of the study period since their treatment was conducted in the course of the last year; therefore, these patients were excluded from analysis. Eight patients with neurofibromatosis were also excluded from analysis. Thus, 557 patients were included in our retrospective study: 452 patients $(81 \%)$ had been treated with radiosurgery alone, whereas 105 patients (19\%) had been treated with combined microsurgery-radiosurgery. In the combined group, microsurgery was performed prior to GKRS. All data were evaluated retrospectively. Patient and tumor characteristics are presented in Table 1 . Koos acoustic neuroma grades were assessed using preoperative and preradiosurgical contrast-enhanced T1- and T2weighted MRI and/or surgical reports. ${ }^{21}$ According to the Koos classification, grade I tumors are purely intracanalicular, grade II neuromas extend into the cerebellopontine angle without contact with the brainstem and have a maximum diameter of $20 \mathrm{~mm}$, grade III neuromas occupy the cerebellopontine cistern without brainstem displacement, and grade IV tumors compress the brainstem and displace cranial nerves. ${ }^{21}$

\section{Radiosurgical Technique}

Patients were treated with a Leksell Gamma Knife (model B until 2011 and Perfexion from 2012 onwards, Elekta AB). The KULA system (Elekta AB) was used as planning software between 1992 and August 1997. GammaPlan (Elekta AB) was used from August 1997 onwards. The MRI planning sequences were performed on a 1.5-T scanner. Gadolinium contrast-enhanced T1-weighted MRI studies and T2-weighted MRI studies, as well as contrast- enhanced and bone window CT scans, were used in the treatment planning. The target was defined as a contrastenhanced tumor mass on T1 sequences and as a hypointense tumor mass on $\mathrm{T} 2$ sequences. The whole tumor mass was covered without an additional margin. The intrameatal treatment volume was adapted according to bone window CT scans. The GKRS parameters are outlined in Table 2. In our pre-modern radiosurgical era (1992-2002), prescribed doses mainly targeted the $50 \%$ isodose line. In our modern radiosurgical era (2003-2016), isodose lines were adapted according to the Koos neuroma grade, although the prescription dose remained 12 Gy for almost all tumors. More specifically, prescribed doses mainly targeted the $70 \%$ isodose line for small intrameatal acoustic neuromas, the $60 \%$ or $70 \%$ isodose line for Koos grade II neuromas, and the $50 \%$ isodose line for Koos grade III or IV tumors. The median cochlear dose was retrospectively and uniformly assessed on bone window CT scans by one investigator (B.G.) using the histogram function of GammaPlan (Elekta $\mathrm{AB}$ ) on the original treatment plans, on several consecutive days. Thus, the median cochlear dose could be retrospectively evaluated in 463 (83\%) of 557 patients.

\section{Follow-Up and Outcome Evaluation}

Data for patients who were lost to follow-up were included in the study but excluded from the outcome analysis. Forty-one (7\%) of 557 patients were lost to follow-up. In addition, we performed a death register comparison using data provided by Statistik Austria; therefore, we were able to determine that $18(3 \%)$ of the 557 patients had died from other causes before a follow-up examination could be conducted. Thus, 498 patients had a minimum followup of 1 year, and 426 patients had a minimum follow-up of 2 years.

Therefore, we report long-term follow-up data on 426 patients with spontaneous acoustic neuroma. The median follow-up after GKRS was 5.1 years (2.0-20.7 years). Twenty-five percent of the patients (106 of 426) presented with a follow-up time equal to or longer than 10 years after radiosurgery. After GKRS, patients were generally followed up with MRI every year for the first 3 years and every 2-5 years in the subsequent follow-up period, depending on the radiological and clinical outcomes. At the same intervals, a clinical evaluation was conducted. However, as is usual in the clinical course, not every patient adhered to this schedule.

Patients were retrospectively assessed according to the Gardner-Robertson (GR) hearing scale ${ }^{14}$ and the HouseBrackmann (HB) facial nerve function scale, ${ }^{17}$ both prior to GKRS and at the time of each follow-up. Thus, hearing function at the last follow-up was rated as preexisting anacusia, hearing deterioration to anacusia, hearing deterioration to nonserviceable hearing, hearing deterioration but still serviceable, or no hearing deterioration. At follow-up, brain MRI studies were evaluated and compared with treatment planning MRI studies, and the status of the treated neuroma was rated as stable, regression, or progression. A progression was diagnosed if the largest tumor diameter had increased by $>20 \%$. A regression was noted if the largest tumor diameter had decreased by $>20 \% .^{15}$ 
TABLE 1. Summary of patient and tumor characteristics

\begin{tabular}{|c|c|c|c|}
\hline Parameter & Combined Approach & GKRS Only & Total \\
\hline Total no. of patients & 105 & 452 & 557 \\
\hline Median age at diagnosis in yrs (range) & $47(16-77)$ & $58(24-84)$ & $57(16-84)$ \\
\hline F/M ratio & $68: 37$ & 260:192 & $328: 229$ \\
\hline \multicolumn{4}{|l|}{ Main neurology at time of diagnosis } \\
\hline Hypacusis & $80(76)$ & $322(71)$ & $402(72)$ \\
\hline Tinnitus & $5(5)$ & $41(9)$ & $46(8)$ \\
\hline Vertigo & $13(12)$ & 50 (11) & $63(11)$ \\
\hline CN VII palsy & $1(1)$ & $3(1)$ & $4(1)$ \\
\hline CN V sensory loss & $2(2)$ & $4(1)$ & $6(1)$ \\
\hline Incidental finding & $1(1)$ & $25(6)$ & $26(5)$ \\
\hline Other & $3(3)$ & $7(2)$ & $10(2)$ \\
\hline \multicolumn{4}{|l|}{ Koos grade at time of GKRS } \\
\hline Grade I & $7(7)$ & $67(15)$ & $74(13)$ \\
\hline Grade II & $24(23)$ & $163(36)$ & $187(34)$ \\
\hline Grade III & $32(30)$ & $126(28)$ & $158(28)$ \\
\hline Grade IV & $42(40)$ & $96(21)$ & $138(25)$ \\
\hline \multicolumn{4}{|l|}{ GR hearing class at time of GKRS } \\
\hline Class I, good & $0(0)$ & $39(9)$ & $39(7)$ \\
\hline Class II, serviceable & $2(2)$ & $147(33)$ & $149(27)$ \\
\hline Class III, nonserviceable & 20 (19) & $155(34)$ & $175(31)$ \\
\hline Class IV, poor & $11(10)$ & $60(13)$ & $71(13)$ \\
\hline Class V, deaf & $70(67)$ & $47(10)$ & $117(21)$ \\
\hline Not sufficiently evaluable & $2(2)$ & $4(1)$ & $6(1)$ \\
\hline \multicolumn{4}{|l|}{ HB grade at time of GKRS } \\
\hline Grade I, normal & $57(54)$ & $435(96)$ & $492(88)$ \\
\hline Grade II, slight dysfunction & $26(25)$ & $10(2)$ & $36(6)$ \\
\hline Grade III, moderate dysfunction & $14(13)$ & $4(1)$ & $18(3)$ \\
\hline Grade IV, moderate to severe dysfunction & $5(5)$ & $3(1)$ & $8(1)$ \\
\hline Grade V, severe dysfunction & $1(1)$ & $0(0)$ & $1(0.2)$ \\
\hline Not sufficiently evaluable & $2(2)$ & $0(0)$ & $2(0.3)$ \\
\hline
\end{tabular}

\section{Statistical Analysis}

Statistical calculations included the Kruskal-Wallis test, Mann-Whitney U-test, and chi-square test for independent samples. In cases of multiple testing, Shaffer's procedure was used as appropriate. Univariate and subsequent multivariate regression analyses were used to assess independent predictors of hearing preservation according to the GR class. The Kaplan-Meier method and actuarial life-table analysis were used to estimate tumor progression and hearing preservation rates. Differences in tumor progression between groups were assessed using the Mantel-Cox and generalized Wilcoxon tests. A p $<0.05$ was considered statistically significant. We used SPSS 23.0 software (SPSS Inc.).

\section{Results}

\section{Patient Presentation and Treatment Era}

When analyzing the different treatment eras, a significant difference became evident. In the 1st decade of radiosurgical treatment at our center (1992-2002), almost half of the patients (57 [43\%] of 133) were treated with microsurgery prior to GKRS, whereas in the modern radiosurgical era (2003-2016), most patients (376 [89\%] of 424) were treated with radiosurgery only $(\mathrm{p}<0.001)$. Of note, the total number of patients in that 1st era of radiosurgical treatment (133 patients) was much smaller than in the second (424). All Koos neuroma grades were treated with GKRS in both the combined approach and the GKRSonly groups. Still, in our pre-modern radiosurgical era, patients were predominantly diagnosed with Koos grade III or IV neuromas (93 [70\%] of 133 patients), whereas in the modern radiosurgical era, significantly fewer patients (203 [48\%] of 424 patients; $p<0.001$ ) were diagnosed with these higher-grade neuromas.

TABLE 2. Gamma Knife radiosurgery parameters

\begin{tabular}{lcc}
\hline \multicolumn{1}{c}{ Parameter } & $\begin{array}{c}\text { Combined } \\
\text { Approach }\end{array}$ & GKRS Only \\
\hline Total no. of patients & 105 & 452 \\
\hline Median vol in $\mathrm{cm}^{3}$ (range) & $2.6(0.2-18.2)$ & $1.2(0.1-19.6)$ \\
\hline Median isodose line in \% (range) & $50(40-80)$ & $50(40-90)$ \\
\hline $\begin{array}{l}\text { Median prescription dose in Gy } \\
\text { (range) }\end{array}$ & $12(10-17)$ & $12(5-15)$ \\
\hline Median central dose in Gy (range) & $24(15-35)$ & $24(10-34)$ \\
\hline
\end{tabular}


TABLE 3. Persisting complications after radiosurgery and subsequent management*

\begin{tabular}{lc}
\hline \multicolumn{1}{c}{ Complication } & No. $(\%)$ \\
\hline None & $466(93.6)$ \\
Hydrocephalus w/o shunt & $3(0.6)$ \\
Hydrocephalus \& shunt & $13(2.6)$ \\
Tumor progression† & $5(1.0)$ \\
Malignant transformationt & $1(0.2)$ \\
Cyst formation \& progression & $1(0.2)$ \\
CN VII paresis persistent & $4(0.8)$ \\
Worsening of preexisting CN VII paresis & $1(0.2)$ \\
Facial tic & $4(0.8)$ \\
\hline
\end{tabular}

* The complication rate includes patients with a minimum follow-up of 1 year (498 patients). Since there was no statistically significant higher complication rate after GKRS in the group of patients that underwent prior microsurgical resection, the table depicts complication data of both groups together. Patients of all treatment eras are included.

$\dagger$ Subsequent microsurgical resection performed.

Clinical presentation at the time of GKRS is described in Table 1. Almost all GKRS targets in the combined approach group (101 [96\%] of 105) were described as residuals after microsurgery. In the remaining 4 cases, patient admission letters describe a recurrence, although postmicrosurgical images were not available. In those cases, microsurgery had been performed prior to 1995 . The majority of patients in the combined group (90 [86\%] of 105) underwent 1 microsurgery prior to GKRS; the remaining patients underwent microsurgery twice (13 [12\%] of 105) or even more often (2 [2\%] of 105). Although a substantial proportion of patients (96 [21\%] of 452) with Koos grade IV neuromas were treated with radiosurgery only, most patients in the combined group (59 [56\%] of 105) were classified as having Koos grade IV neuromas prior to microsurgery ( $\mathrm{p}<0.001)$. However, after microsurgery and before GKRS, $40 \%$ of patients (42 of 105) were still classified as having Koos grade IV neuromas. The median time to GKRS after the last microsurgery was 3.3 years $(0.2-28.6$ years).

\section{Complications After Radiosurgery}

After GKRS, the total rate of persisting complications among the 498 patients with a minimum follow-up of 1 year was $6.4 \%$ (Table 3 ). This rate includes 5 patients who underwent microsurgical resection after GKRS due to (pseudo-) progression of the tumor, as well as 1 patient with malignant transformation (Figs. 1 and 2) after GKRS. Of note, the complication rate in the modern radiosurgical era at our center was significantly $(p=0.039)$ lower $(5 \%)$ than that in the pre-modern era (11\%). A further decline in the complication rate to $4 \%$ has been noted since the implementation of the Gamma Knife Perfexion unit in 2012. Moreover, no persisting new facial nerve paresis was seen after GKRS in our modern radiosurgical era. Hydrocephalus after GKRS was diagnosed in 16 patients (3\% of 498). However, the risk of developing hydrocephalus after GKRS varied significantly among the Koos neuroma grades $(\mathrm{p}<0.001)$. No hydrocephalus was observed among the patients with Koos grade I acoustic neuromas.
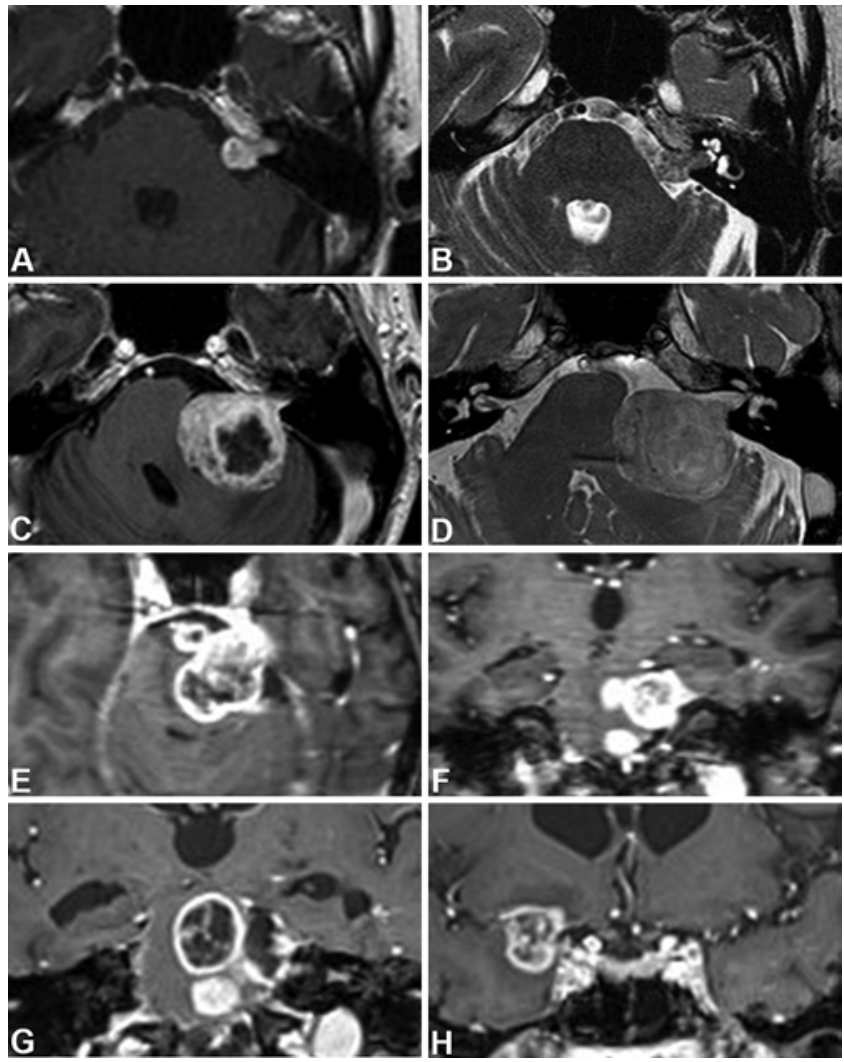

FIG. 1. Images from a case with malignant transformation after GKRS. Planning T1-weighted contrast-enhanced (A) and T2-weighted (B) MRI sequences obtained in a patient undergoing radiosurgery with a 13-Gy prescription dose targeting the $50 \%$ isodose line for acoustic neuroma. A preradiosurgery biopsy was not performed. Axial T1-weighted contrast-enhanced (C) and T2-weighted (D) images obtained 8 years after radiosurgery, showing significant tumor progression. The patient underwent microsurgery. Postoperative MRI showed total tumor resection (not shown). Histopathological analysis revealed schwannoma with a rather high MIB-1 labeling index (see Fig. 2A-D). Eight months later, the patient developed sudden hemiparesis and ataxia. Contrast-enhanced T1-weighted MRI (E and F) revealed a massive, multifocal tumor recurrence. A second microsurgical resection/mass reduction was performed. Histopathology revealed a malignant peripheral nerve sheath tumor (PNST). One month later, follow-up contrast-enhanced T1-weighted MR images $(\mathbf{G}$ and $\mathbf{H})$ revealed another multifocal and even contralateral tumor recurrence and progression.

The hydrocephalus risk was $0.6 \%$ for patients with Koos grade II neuromas, $2.8 \%$ for those with Koos grade III, and $9.1 \%$ for those with Koos grade IV. Although slightly higher in percentage, the complication rate following GKRS did not increase statistically significantly in the group of patients who had undergone prior microsurgical resection.

Separate from the persisting complications, temporary complications were noted as well, including facial tics (13 [3\%] of 498), facial nerve paresis (2 [0.4\%] of 498), and disabling vertigo (1 [0.2\%] of 498) in patients who showed remission after a certain follow-up period. A temporary and generally short-lived worsening of preexisting symptoms, especially vertigo and tinnitus, typically 6 months after GKRS, was reported by a third of the patients (150 [30\%] of 498). 


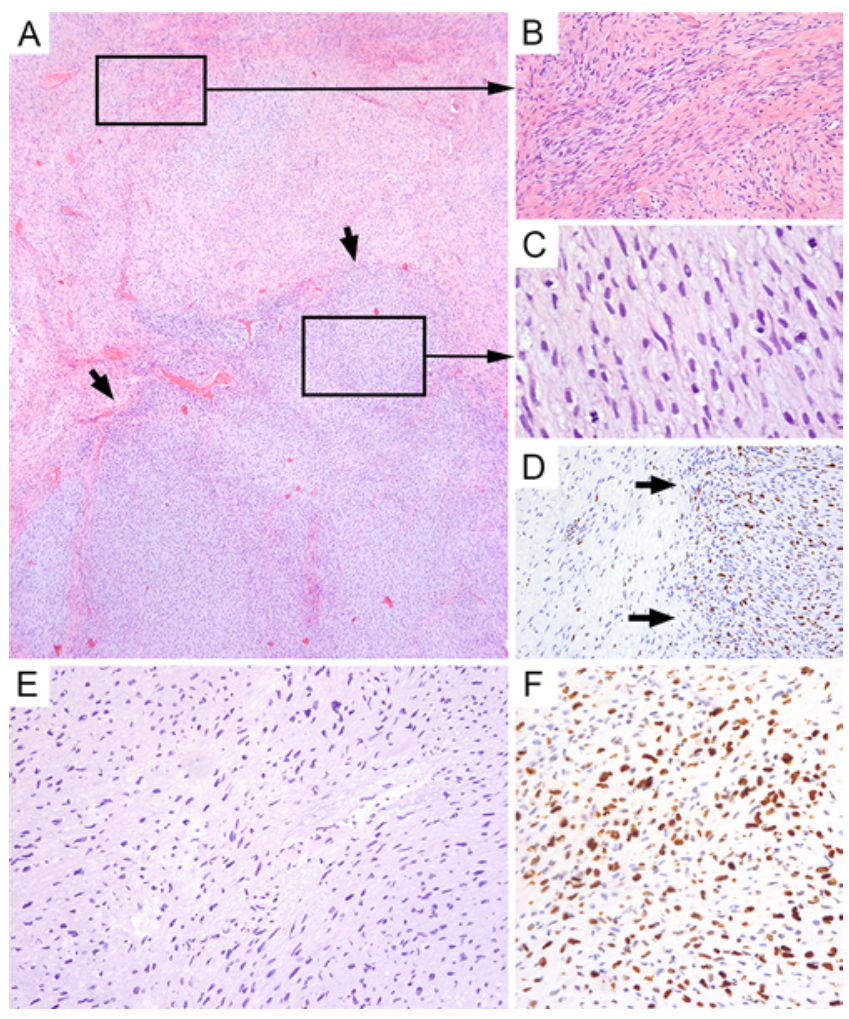

FIG. 2. Histopathological studies from a case with malignant transformation after GKRS. Eight years after GKRS, microsurgical resection and subsequent histopathological analysis revealed a schwannoma (A and inset $\mathbf{B}$ ) but already containing well-delineated areas (A and D, arrows) with increased cellularity, brisk mitotic activity (inset $\mathrm{C}$ ), and a rather high MIB-1 labeling index of 35\%, in retrospect indicating transformation into a malignant PNST. A second microsurgical resection was performed 8 months after the first because of clinical deterioration and multifocal recurrence. Histopathological analysis showed increased anisomorphism throughout the entire tumor tissue $(\mathrm{E})$ with an MIB-1 labeling index of up to $63 \%(\mathbf{F})$, thus fulfilling the criteria for a malignant PNST. One month later the patient died as a result of brainstem compression. $H \& E(A-C, E)$, original magnification $\times 40(A), \times 200(B, D-F), \times 600(C)$. Figure is available in color online only.

\section{Tumor Control and Outcome}

Follow-up and outcome data were available for $426 \mathrm{pa}-$ tients with a minimum follow-up of 2 years. The median follow-up among these patients was 5.1 years, and a quarter of the patients (106 of 426) had a follow-up equal to or longer than 10 years. In total, 2871 patient-years of followup were assessed.

The radiological outcome revealed that the majority of patients were diagnosed with stable (220 [52\%] of 426) or shrinking (174 [41\%] of 426) tumors. Thus, we report an overall tumor control rate of $92 \%$ at the last followup. Actuarial statistics are provided in Fig. 3A. When the radiosurgery-only and combined groups were analyzed separately, no difference in tumor control rates was revealed. Twenty-three patients (5\% of 426) showed initial tumor progression or swelling after radiosurgical treatment, but the tumors stabilized on their own after several years of follow-up. Thus, there was a $98 \%$ rate of overall tumor control without the need for additional treatment at the last follow-up. Actuarial statistics also revealed a tumor control rate with no additional treatment of $98 \%$ at $5,10,15$, and 20 years. In only 6 patients $(1.4 \%$ of 426$)$ was microsurgical resection after GKRS considered necessary because of tumor growth or the patient's wishes. In 1 of these cases, a malignant peripheral nerve sheath tumor was diagnosed histopathologically (Figs. 1 and 2). Furthermore, 3 patients (1\% of 426) had clinical follow-up but no radiological follow-up. The radiological outcome showed no significant association either with the Koos neuroma grade at the time of GKRS or with prior microsurgical resection. Of note, the rate of stable or regressing lesions among Koos grade IV acoustic neuromas was similar to that for all other Koos grades (Figs. 3B and 4). Two of the 6 patients who underwent microsurgical resection after GKRS had initially been diagnosed with Koos grade IV acoustic neuroma.

Over the course of the last 25 years at our center, several prescription doses have been applied to treat acoustic neuromas. Significant differences were found between the pre-modern and the modern radiosurgical eras $(\mathrm{p}<0.001)$ in terms of the prescribed dose: Since 2002, most patients (and since 2012, almost all patients) have been treated with a prescription dose of 12 Gy. In earlier years, however, a prescription dose of 13 or 14 Gy was regularly used as well. Note that when we pooled these doses by groups of patients who had received below 12 Gy, exactly 12 Gy, and above $12 \mathrm{~Gy}$, we found no statistically significant impact on the radiological outcome.

We additionally performed univariate log-rank tests of time to progression and the impact of radiosurgical dose, Koos neuroma grade, and microsurgical pretreatment. No significant difference in tumor control was found; thus, further multivariate regression analysis was not performed.

\section{Hearing Preservation and Outcome-Influencing Risk Factors}

Among all patients with a minimum follow-up of 2 years, univariate regression analysis revealed that the GR hearing class prior to GKRS, Koos grade at GKRS, median cochlear dose, age at GKRS, prescription dose, radiation time, and target volume seemed to have a significant impact on the GR hearing class at the last follow-up. However, in the multivariate regression model, only the GR class prior to GKRS and the median dose to the cochlea were found to be independent predictors of the GR class at follow-up ( $<<0.001$ and $p=0.029$, respectively).

The number of patients with nonserviceable or worse hearing prior to GKRS was generally rather high (Table 1). Moreover, in our pre-modern radiosurgical era, most patients $(79 \%)$ presented with already nonserviceable or worse hearing at the time of GKRS. In contrast, in the last decade, more than a third of the patients (39\%) were diagnosed with still-serviceable or good hearing.

Thus, 132 patients who were diagnosed with good or serviceable hearing prior to GKRS, had no additional treatment after GKRS, and had a minimum follow-up of 2 years can be included in the following subgroup analysis. Among these patients, the rate of serviceable hearing at the last follow-up was 55\%. We aimed to evaluate a cutoff value for the median cochlear dose by pooling cochlear 

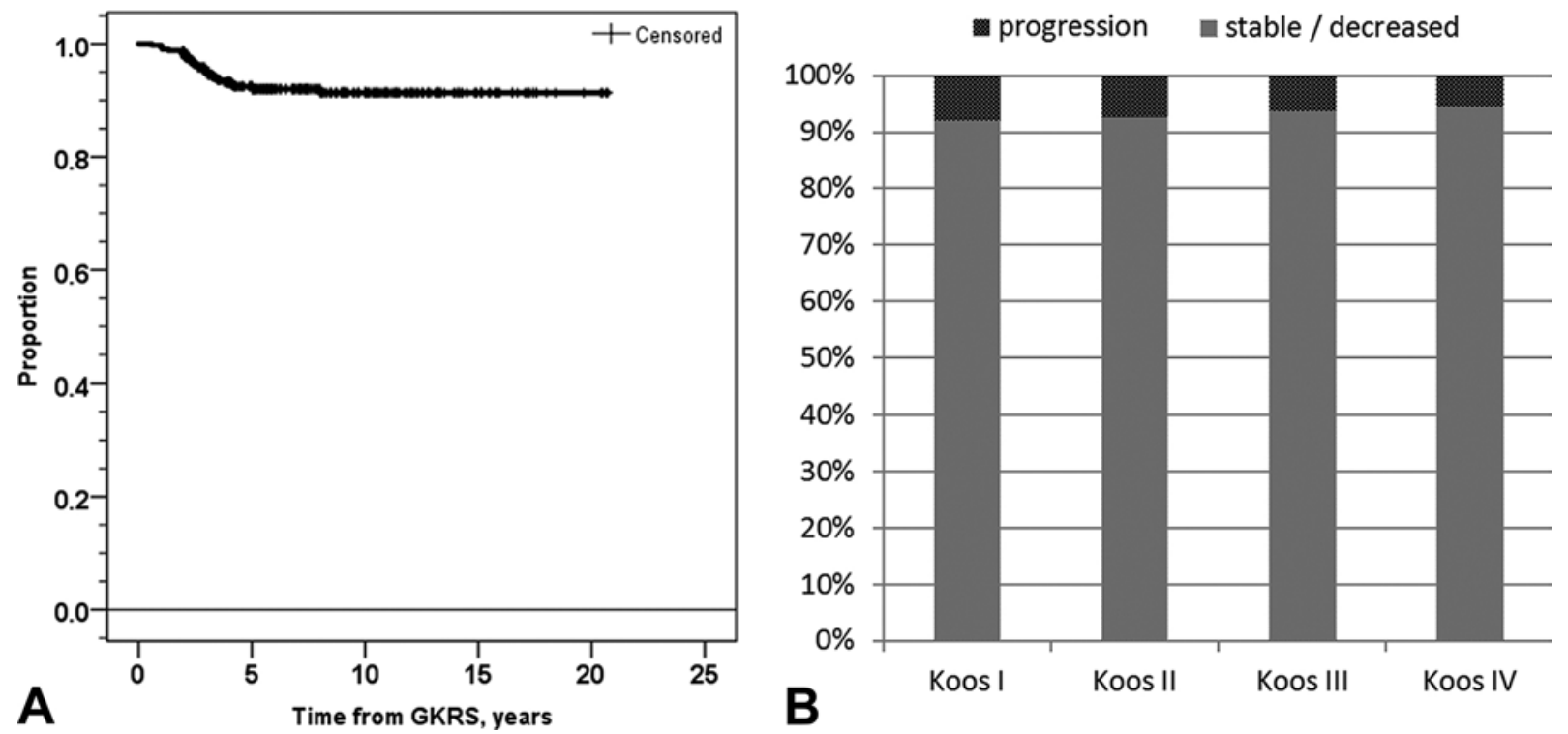

FIG. 3. A: Kaplan-Meier analysis of the tumor control rate in a sample with a minimum of 2 years of follow-up (423 patients). Patients were censored at the last available radiological follow-up. Time is relative to the date of GKRS. Actuarial analysis revealed radiological tumor control rates of $92 \%, 91 \%, 91 \%$, and $91 \%$ at $5,10,15$, and 20 years, respectively. B: Bar graph of tumor control rates according to Koos acoustic neuroma grades. Tumor control rates were similar across all Koos grades.

doses above and below 4 Gy, 5 Gy, and 6 Gy. In our series, after correction for multiple testing, patients who received a median cochlear dose above 6 Gy showed a significantly higher rate of nonserviceable hearing (pooled GR classes III-V) at the last follow-up than that in patients in whom the median cochlear dose was retrospectively evaluated as less than $6 \mathrm{~Gy}(\mathrm{p}=0.027)$. Actuarial statistics revealed hearing preservation rates of $53 \%, 34 \%$, and $34 \%$ at 5,10 , and 15 years after GKRS among the 132 patients. Four of the patients were excluded from these actuarial statistics: Although recent follow-up data indicating nonserviceable hearing were available, the exact date of the loss of serviceable hearing could not be determined since these patients did not regularly show up for their follow-up appointments.

\section{Discussion}

\section{The Ever-Changing Landscape in Acoustic Neuroma Management}

Over the last decades, the management of acoustic neuroma has changed. Microsurgery, formerly recognized as the primary treatment for all tumor grades, has increasingly been replaced by stereotactic radiosurgery (SRS) ${ }^{8,30-32}$ Excellent tumor control rates following SRS have been widely reported and range from $87 \%$ to $100 \%{ }^{7,16,30,33,35}$ In a recent publication, Kondziolka and colleagues suggested that the only remaining indications for resection in a patient with a small to moderate-sized vestibular schwannoma are brainstem compression, intractable trigeminal neuralgia or headache, hydrocephalus, an unclear diagnosis, and the patient's choice..$^{22} \mathrm{We}$ have made similar observations about the last 25 years of treatment provided at our institution: Since 2002, the majority of GKRS-treated acoustic neuromas have been treated with radiosurgery alone, whereas before 2002, pa- tients were often treated with combined microsurgery-radiosurgery. The shift toward radiosurgery becomes even more apparent when analyzing our modern radiosurgical treatment era since the implementation of the Gamma Knife Perfexion unit: 352 patients with a diagnosis of acoustic neuroma were treated at our department between 2012 and 2016. Almost two-thirds of those patients underwent radiosurgery alone, and only one-third primarily underwent microsurgical resection, though some were later treated radiosurgically as well. Unfortunately, not all issues regarding acoustic neuroma treatment have been resolved. Indeed, a recent extensive study on the natural course of acoustic neuroma reported that, after a median follow-up of less than 2 years, more than $40 \%$ of patients presented with tumor growth, thus validating early treatment in our opinion. ${ }^{19}$

In a recently published analysis of the Surveillance, Epidemiology, and End Results (SEER) database, authors from the US reported that increasing numbers of patients are treated conservatively. ${ }^{8}$ However, other studies have indicated that a wait-and-see policy exposes patients to elevated risks of tumor growth and degradation of hearing. ${ }^{19,32,33,42}$

This shift toward conservatism described in some recent studies is counterbalanced by other equally recent studies in which it is clearly noted that-besides agepatient race and insurance status seem to independently influence acoustic neuroma management and outcome. ${ }^{4,26}$ Many single-center studies and meta-analyses have evaluated both microsurgery and radiosurgery as effective treatment options for acoustic neuroma. $37,7,11,22,23,32,33,35,36,41$ The tumor control rates in our study match the excellent control rates following radiosurgery that are amply described in the literature. ${ }^{7,16,33,35}$ Moreover, for small to medium-sized neuromas, radiosurgery has been reported to have clear advantages over microsurgery in terms of post- 

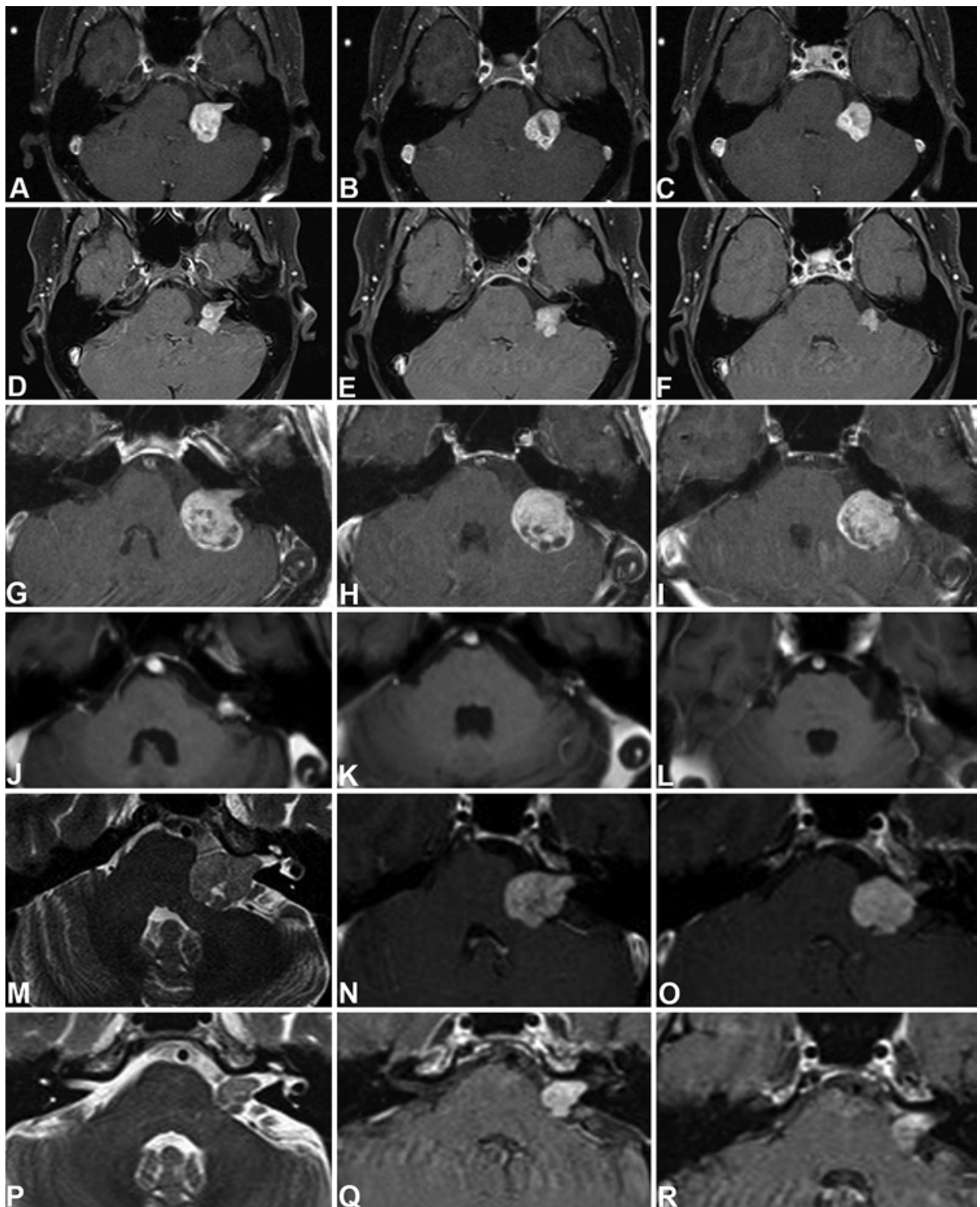

FIG. 4. Examples of patients with larger acoustic neuromas and their long-term follow-ups. Three months after initial patient presentation with tinnitus and hearing deterioration, an acoustic neuroma was radiologically diagnosed on contrast-enhanced T1weighted MR images (A-C). At the time of GKRS, the patient had GR class II hearing. A prescription dose of 12 Gy was delivered to the $50 \%$ isodose line. The target volume was $4.7 \mathrm{~cm}^{3}$. Ten-year follow-up contrast-enhanced T1-weighted MRI showed significant tumor shrinkage (D-F). At the time of the last follow-up, the patient was experiencing nonserviceable hearing (GR class III) but had grown accustomed to the persisting tinnitus. No postradiosurgical complications were noted. Another patient experienced nonserviceable hearing (GR class III) and slight dysfunction of the facial nerve (HB grade II). Contrast-enhanced T1-weighted MRI (G-I) showed an acoustic neuroma with slight compression of the brainstem, which was radiosurgically treated in 1999. A prescription dose of $12 \mathrm{~Gy}$ was delivered to the $50 \%$ isodose line. The target volume was $9.1 \mathrm{~cm}^{3}$. Regular follow-up MRI showed continuous tumor shrinkage. At the 17-year follow-up, the tumor volume was significantly reduced $(\mathrm{J}-\mathrm{L})$. The patient experienced poor hearing (GR class IV), but no postradiosurgical complications had occurred. A third patient initially presented with nonserviceable hearing (GR class III). Contrast-enhanced T1-weighted MRI (M-0) showed an acoustic neuroma with moderate compression of the brainstem. A prescription dose of $12 \mathrm{~Gy}$ was delivered to the $45 \%$ isodose line. The target volume was $3.4 \mathrm{~cm}^{3}$. After GKRS, the patient experienced a transient tic and transient facial paresis. The patient received dexamethasone treatment and was relieved from both symptoms a few months after GKRS. At the 8-year follow-up, MRI (P-R) showed a significant reduction in tumor volume. The patient's hearing was graded as GR class III at follow-up.

interventional complication rates and reduced costs for patients and the health care system..$^{12,22,27,41}$ Adding to the body of evidence is an extensive multicenter study reporting that quality of life is rather similar between manage- ment options and is affected by the diagnosis of acoustic neuroma rather than the treatment strategy. ${ }^{11}$ Other studies have even attributed a higher quality of life to radiosurgically treated patients. ${ }^{30}$ 


\section{Evolution of Radiosurgical Treatment Planning and Postradiosurgical Complications}

Hardware and software improvements over the past decades have significantly changed the radiosurgical field. When we started GKRS treatment of acoustic neuroma in the early 1990s, treatment planning focused on the $50 \%$ isodose line. Typical prescription doses at our center and elsewhere were 13 and 14 Gy or even higher. ${ }^{13}$ Soon thereafter, at least at our center, prescription doses of 12 or 13 Gy replaced the higher prescription doses. In our modern radiosurgical era, we almost exclusively treated acoustic neuromas with a 12-Gy prescription dose. According to our outcome data, tumor control rates among patients treated with a 12-Gy prescription dose are equal to those among patients treated with higher prescription doses. Moreover, isodose lines are now adapted according to the Koos neuroma grade: prescribed doses mainly target the $70 \%$ isodose line for small intrameatal acoustic neuromas, the $60 \%$ or $70 \%$ isodose line for Koos grade II tumors, and the 50\% isodose line for Koos grades III or IV tumors. For larger acoustic neuromas treated on the $50 \%$ isodose line, planning is conducted in such a way that the high dose range is as small as possible and out of the way of the presumed course of the facial nerve. Because of this treatment strategy, complication rates in our modern radiosurgical era are significantly lower than before. Moreover, no persisting facial nerve paresis after GKRS was seen in our modern radiosurgical era. Furthermore, we have aimed to minimize the cochlear dose, which has been significantly facilitated since the implementation of the Gamma Knife Perfexion unit in 2012 and the option of dynamic shaping. Still, we do not recommend undertreating intracanalicular tumors in favor of low cochlear doses. ${ }^{20}$

Radiosurgery as a treatment option for large acoustic neuromas remains a matter of controversy. In our series, a rather high number of patients were diagnosed with acoustic neuromas classified as Koos grade IV and showed good outcomes. Long-term tumor control rates were similar to those observed for other Koos neuroma grades. However, larger acoustic neuromas in our series mainly presented with minimal or moderate brainstem compression. Still, complication rates were higher among the patients with large acoustic neuromas. There was a significant risk of developing hydrocephalus after GKRS, even in our modern radiosurgical era, whereas, at least in our series, other complications were comparable to those observed for other Koos neuroma grades. ${ }^{6,16}$ Radiosurgery has been described as an effective management option for patients whose tumors remain or recur after microsurgery. ${ }^{17}$ In our series, we found high tumor control rates after a combined microsurgical-radiosurgical treatment as well. Thus, for large or giant acoustic neuromas with significant brainstem compression or hydrocephalus, the following combined approach should be considered: initial microsurgical brainstem decompression with functional and anatomical preservation of the neural structures, followed by radiosurgery for residual tumor. ${ }^{18,22}$

\section{Limitations of Our Study}

Our study reports on long-term follow-up data in a large series of acoustic neuroma patients. Limitations of our study include its retrospective character as well as its center and treatment bias. Because of our hospital's treatment policy, we cannot provide comprehensive data on the natural history of acoustic neuromas. However, our median follow-up of 5 years and range of 2-21 years after radiosurgery are among the longest reported follow-up periods. Moreover, 106 patients $(25 \%)$ had follow-up times equal to or longer than 10 years after radiosurgery.

\section{Malignant Transformation After Radiosurgery}

In radiosurgical treatment, the irradiated volume is generally small and the high single doses administered to the target should theoretically lead to cytotoxicity and not mutagenicity ${ }^{28}$ Generally, the risk of a malignancy developing after radiosurgery has been specified as $0.04 \%$ at 15 years after treatment. ${ }^{28}$ Single cases of malignant transformation after radiosurgical management of acoustic neuromas have been reported in large series, with a calculated risk between $0.03 \%$ and $0.3 \% .^{15,24,28}$ However, malignant transformation of acoustic neuroma has been described after microsurgery as well. ${ }^{24}$ In our series, 1 case of malignant transformation of a spontaneous acoustic neuroma was observed, amounting to a risk rate of $0.2 \%$ (Figs. 1 and 2). The malignant transformation was found 8 years after GKRS. No preradiosurgery biopsy was available in this patient; thus, neither Cahan's criteria to determine if secondary neoplasms are radiation induced nor Cahan's criteria as modified per Maducdoc et al. were fulfilled. ${ }^{24}$ Moreover, de novo malignant peripheral nerve sheath tumors of the eighth cranial nerve have been reported without prior irradiation as well. ${ }^{9}$ Taking all of these factors into consideration, we assert that GKRS is a safe treatment option for acoustic neuroma patients, but caution is necessary when counseling young patients and patients with neurofibromatosis. ${ }^{9,15,24,28}$

\section{Hearing Preservation and Dose to the Cochlea}

To quote a recent paper by Jacob and colleagues, "it is not yet clear if a reliable hearing preservation is the "holy grail' or the 'unicorn' in acoustic neuroma treatment." 20 Declines in hearing have been described in a significant portion of patients after relatively short follow-ups, even among patients who only had intrameatal acoustic neuromas and went without treatment. ${ }^{29,32}$ Similar to our data, among patients with serviceable hearing prior to GKRS, the rate of serviceable hearing preservation following radiosurgery is generally reported to be approximately $50 \%$ at the long-term follow-up and is sometimes reported to be higher. ${ }^{15,23,32,37}$ Tumor size, cochlear dose, age, initial symptom other than hearing decrement, and level of hearing prior to treatment have been described as risk factors. ${ }^{20,23,37}$ Our data indicate that the level of hearing prior to treatment and the cochlear dose are the main risk factors among patients with serviceable hearing prior to treatment. In our opinion, it is important to note that the majority of patients generally present with nonserviceable hearing prior to GKRS. Although we try to minimize the cochlear dose, we, like the authors of a previously published paper, do not recommend undertreating intracanalicular tumors in favor of low cochlear doses since several other factors seem to play crucial roles. ${ }^{20}$ 
In recent small series of NF2 patients with postlingual deafness, cochlear implants (CIs) have provided favorable hearing restoration..$^{39,40} \mathrm{In}$ addition, several new aspects in the context of CIs have apparently reopened the discussion on the management of both sporadic and NF2-associated acoustic neuromas. Cochlear implants have been shown to restore hearing and suppress tinnitus even in patients with contralateral normal hearing. ${ }^{2}$ Modern CIs seem to allow postimplantation MRI and the depiction of the ipsilateral internal auditory canal and cerebellopontine angle. ${ }^{37,38}$ Moreover, electrophysiological assessment of the auditory nerve has become more reliable in predicting the nerve's integrity and thus the possibility to restore hearing with CIs. ${ }^{5}$ The latter point is of importance in the context of GKRS: one topic of discussion is that radiation-induced injury to the cochlear nerve would compromise the potential for hearing restoration through a CI and that radiosurgery might be disadvantageous for long-term preservation or potential restoration of hearing. ${ }^{40}$ To date, only circumstantial evidence exists regarding CIs and radiosurgery or radiotherapy. However, available data show that CIs lead to improved hearing in NF2 patients who have undergone radiation treatment to control their acoustic neuroma. ${ }^{34,39}$ In the future, hearing remediation among patients with spontaneous acoustic neuroma may also include cochlear implantation-all discussion about costs to the patients and the health care system and possible complications aside. ${ }^{1,32,34}$

\section{Conclusions}

Radiosurgery for acoustic neuroma is safe and effective. Improvements in treatment planning have further reduced the risk of complications and maintained excellent tumor control rates. In small to medium-sized spontaneous acoustic neuromas, radiosurgery should be recognized as the primary treatment at an early stage. Although the cochlear dose should be minimized, undertreating intracanalicular tumors in favor of low cochlear doses is not recommended. For large acoustic neuromas, radiosurgery remains a reliable management option with tumor control rates similar to those for smaller neuromas; however, careful patient selection and counseling are recommended because of the higher risk of side effects. Microsurgery must be considered in acoustic neuromas with significant brainstem compression or hydrocephalus. Effective tumor control rates can be achieved by combined microsurgicalradiosurgical management, which includes partial microsurgical resection with functional and anatomical preservation of the neural structures and subsequent radiosurgery for residual tumor.

\section{References}

1. Akpinar B, Mousavi SH, McDowell MM, Niranjan A, Faraji $\mathrm{AH}$, Flickinger JC, et al: Early radiosurgery improves hearing preservation in vestibular schwannoma patients with normal hearing at the time of diagnosis. Int J Radiat Oncol Biol Phys 95:729-734, 2016

2. Arnoldner C, Lin VY: Expanded selection criteria in adult cochlear implantation. Cochlear Implants Int 14 (Suppl 4):S10-S13, 2013

3. Arthurs BJ, Fairbanks RK, Demakas JJ, Lamoreaux WT,
Giddings NA, Mackay AR, et al: A review of treatment modalities for vestibular schwannoma. Neurosurg Rev 34:265279, 2011

4. Babu R, Sharma R, Bagley JH, Hatef J, Friedman AH, Adamson C: Vestibular schwannomas in the modern era: epidemiology, treatment trends, and disparities in management. J Neurosurg 119:121-130, 2013

5. Bahmer A, Peter O, Baumann U: Recording of electrically evoked auditory brainstem responses (E-ABR) with an integrated stimulus generator in Matlab. J Neurosci Methods 173:306-314, 2008

6. Bailo M, Boari N, Franzin A, Gagliardi F, Spina A, Del Vecchio A, et al: Gamma Knife radiosurgery as primary treatment for large vestibular schwannomas: clinical results at long-term follow-up in a series of 59 patients. World Neurosurg 95:487-501, 2016

7. Boari N, Bailo M, Gagliardi F, Franzin A, Gemma M, del Vecchio A, et al: Gamma Knife radiosurgery for vestibular schwannoma: clinical results at long-term follow-up in a series of 379 patients. J Neurosurg 121 Suppl:123-142, 2014

8. Carlson ML, Habermann EB, Wagie AE, Driscoll CL, Van Gompel JJ, Jacob JT, et al: The changing landscape of vestibular schwannoma management in the United States - a shift toward conservatism. Otolaryngol Head Neck Surg 153:440-446, 2015

9. Carlson ML, Jacob JT, Habermann EB, Glasgow AE, Raghunathan A, Link MJ: Malignant peripheral nerve sheath tumors of the eighth cranial nerve arising without prior irradiation. J Neurosurg 125:1120-1129, 2016

10. Carlson ML, Link MJ, Wanna GB, Driscoll CL: Management of sporadic vestibular schwannoma. Otolaryngol Clin North Am 48:407-422, 2015

11. Carlson ML, Tveiten OV, Driscoll CL, Goplen FK, Neff BA, Pollock BE, et al: Long-term quality of life in patients with vestibular schwannoma: an international multicenter crosssectional study comparing microsurgery, stereotactic radiosurgery, observation, and nontumor controls. J Neurosurg 122:833-842, 2015

12. Caruso JP, Moosa S, Fezeu F, Ramesh A, Sheehan JP: A cost comparative study of Gamma Knife radiosurgery versus open surgery for intracranial pathology. J Clin Neurosci 22:184-188, 2015

13. Flickinger JC, Kondziolka D, Niranjan A, Maitz A, Voynov G, Lunsford LD: Acoustic neuroma radiosurgery with marginal tumor doses of 12 to 13 Gy. Int J Radiat Oncol Biol Phys 60:225-230, 2004

14. Gardner G, Robertson JH: Hearing preservation in unilateral acoustic neuroma surgery. Ann Otol Rhinol Laryngol 97:55-66, 1988

15. Hasegawa T, Kida Y, Kato T, Iizuka H, Kuramitsu S, Yamamoto T: Long-term safety and efficacy of stereotactic radiosurgery for vestibular schwannomas: evaluation of 440 patients more than 10 years after treatment with Gamma Knife surgery. J Neurosurg 118:557-565, 2013

16. Hasegawa T, Kida Y, Kobayashi T, Yoshimoto M, Mori Y, Yoshida J: Long-term outcomes in patients with vestibular schwannomas treated using gamma knife surgery: 10-year follow up. J Neurosurg 102:10-16, 2005

17. House JW, Brackmann DE: Facial nerve grading system. Otolaryngol Head Neck Surg 93:146-147, 1985

18. Huang MJ, Kano H, Mousavi SH, Niranjan A, Monaco EA III, Arai Y, et al: Stereotactic radiosurgery for recurrent vestibular schwannoma after previous resection. J Neurosurg 126:1506-1513, 2017

19. Hunter JB, Francis DO, O'Connell BP, Kabagambe EK, Bennett ML, Wanna GB, et al: Single institutional experience with observing 564 vestibular schwannomas: factors associated with tumor growth. Otol Neurotol 37:1630-1636, 2016

20. Jacob JT, Carlson ML, Schiefer TK, Pollock BE, Driscoll 
CL, Link MJ: Significance of cochlear dose in the radiosurgical treatment of vestibular schwannoma: controversies and unanswered questions. Neurosurgery 74:466-474, 2014

21. Koos WT, Spetzler RF, Lang J: Color Atlas of Microsurgery. New York: Thieme, 1993, pp 530-532

22. Kondziolka D, Shin SM, Brunswick A, Kim I, Silverman JS: The biology of radiosurgery and its clinical applications for brain tumors. Neuro Oncol 17:29-44, 2015

23. Lunsford LD, Niranjan A, Flickinger JC, Maitz A, Kondziolka D: Radiosurgery of vestibular schwannomas: summary of experience in 829 cases. J Neurosurg 102 Suppl:195-199, 2005

24. Maducdoc MM, Ghavami Y, Linskey ME, Djalilian HR: Evaluation of reported malignant transformation of vestibular schwannoma: de novo and after stereotactic radiosurgery or surgery. Otol Neurotol 36:1301-1308, 2015

25. Marston AP, Jacob JT, Carlson ML, Pollock BE, Driscoll CLW, Link MJ, et al: Pretreatment growth rate as a predictor of tumor control following Gamma Knife radiosurgery for sporadic vestibular schwannoma. J Neurosurg 127:380-387, 2017

26. McClelland S III, Guo H, Okuyemi KS: Morbidity and mortality following acoustic neuroma excision in the United States: analysis of racial disparities during a decade in the radiosurgery era. Neuro Oncol 13:1252-1259, 2011

27. Myrseth E, Møller P, Pedersen PH, Lund-Johansen M: Vestibular schwannoma: surgery or gamma knife radiosurgery? A prospective, nonrandomized study. Neurosurgery 64:654-663, 2009

28. Nicolli EA, Ruckenstein M: What is the risk of malignant transformation of vestibular schwannoma following radiosurgery? Laryngoscope 125:1761-1762, 2015

29. Pennings RJ, Morris DP, Clarke L, Allen S, Walling S, Bance ML: Natural history of hearing deterioration in intracanalicular vestibular schwannoma. Neurosurgery 68:68-77, 2011

30. Pollock BE, Driscoll CL, Foote RL, et al: Patient outcomes after vestibular schwannoma management: a prospective comparison of microsurgical resection and stereotactic radiosurgery. Neurosurgery 59:77-85, 2006

31. Pollock BE, Lunsford LD, Norén G: Vestibular schwannoma management in the next century: a radiosurgical perspective. Neurosurgery 43:475-483, 1998

32. Régis J, Carron R, Park MC, Soumare O, Delsanti C, Thomassin JM, et al: Wait-and-see strategy compared with proactive Gamma Knife surgery in patients with intracanalicular vestibular schwannomas. J Neurosurg 113 Suppl:105-111, 2010

33. Régis J, Pellet W, Delsanti C, Dufour H, Roche PH, Thomassin JM, et al: Functional outcome after gamma knife surgery or microsurgery for vestibular schwannomas. J Neurosurg 97:1091-1100, 2002

34. Roehm PC, Mallen-St Clair J, Jethanamest D, Golfinos JG, Shapiro W, Waltzman S, et al: Auditory rehabilitation of patients with neurofibromatosis Type 2 by using cochlear implants. J Neurosurg 115:827-834, 2011

35. Rowe JG, Radatz MW, Walton L, Hampshire A, Seaman S, Kemeny AA: Gamma knife stereotactic radiosurgery for uni- lateral acoustic neuromas. J Neurol Neurosurg Psychiatry 74:1536-1542, 2003

36. Samii M, Gerganov V, Samii A: Hearing preservation after complete microsurgical removal in vestibular schwannomas. Prog Neurol Surg 21:136-141, 2008

37. Tamura M, Carron R, Yomo S, Arkha Y, Muraciolle X, Porcheron D, et al: Hearing preservation after gamma knife radiosurgery for vestibular schwannomas presenting with high-level hearing. Neurosurgery 64:289-296, 2009

38. Todt I, Rademacher G, Mittmann P, Mutze S, Ernst A: Postoperative imaging of the internal auditory canal. Visualization of active auditory implants. HNO 65 (Suppl 2):S81-S86, 2017

39. Trotter MI, Briggs RJ: Cochlear implantation in neurofibromatosis type 2 after radiation therapy. Otol Neurotol 31:216-219, 2010

40. Upadhyay U, Almefty RO, Dunn IF, Al-Mefty O: Save the nerve. J Neurosurg 123:821-823, 2015 (Letter)

41. Wolbers JG, Dallenga AH, Mendez Romero A, van Linge A: What intervention is best practice for vestibular schwannomas? A systematic review of controlled studies. BMJ Open 3:e001345, 2013

42. Yamakami I, Uchino Y, Kobayashi E, Yamaura A: Conservative management, gamma-knife radiosurgery, and microsurgery for acoustic neurinomas: a systematic review of outcome and risk of three therapeutic options. Neurol Res 25:682-690, 2003

\section{Disclosures}

None of the authors disclosed any competing interests or specific funding regarding this retrospective study.

\section{Author Contributions}

Conception and design: Gatterbauer, Frischer. Acquisition of data: Gatterbauer, Frischer, Gruber, Schoeffmann, Ertl, Hoeftberger, Mallouhi, Wolfsberger, Eisner, Kitz. Analysis and interpretation of data: Frischer, Kitz. Drafting the article: Gatterbauer, Frischer, Hoeftberger, Arnoldner, Eisner, Knosp. Critically revising the article: Gatterbauer, Frischer, Gruber, Schoeffmann, Hoeftberger, Mallouhi, Wolfsberger, Arnoldner, Eisner, Knosp, Kitz. Reviewed submitted version of manuscript: all authors. Approved the final version of the manuscript on behalf of all authors: Gatterbauer. Statistical analysis: Frischer. Administrative/technical/material support: Ertl. Study supervision: Frischer.

\section{Supplemental Information}

\section{Previous Presentations}

Portions of this work were presented in abstract form/oral presentation at the OEGNC, Linz, Austria, in October 2016 and at the ISRS, Montreux, Switzerland, in May 2017.

\section{Correspondence}

Brigitte Gatterbauer: Medical University Vienna, Austria. brigitte. gatterbauer@meduniwien.ac.at. 